\title{
Airbags for motorcycles situated on the tank
}

\author{
M. Hönig*, J. Kovanda, J. First \\ Department of Transporting Technology, Czech Technical University in Prague, Czech Republic \\ * Corresponding author: xhonig@.fd.cvut.cz
}

\begin{abstract}
The aim of the study is to evaluate the tank as a potential place for motorcycle airbags. The study compiles the efforts of the CTU in Prague, HTW Dresden, as well as JAWA and DELPHI, for the improvement of the passive safety of motorcycle riders. The tank shapes are taken into consideration, as to their effects on passive safety, including their suitability or unsuitability. The possibilities of the software MADYMO as a tool for an initial blue-print of any motorcycle airbag if placed on the tank are also described.
\end{abstract}

KEY WORDS: Passive safety, crash test, airbag for motorcycle, tank deformation, tank shape, computer simulation, use of software MADYMO

\section{INTRODUCTION}

Since 1981 the airbag has become a standard piece of equipment for passenger cars. It has been offered by Honda for some of their motorcycles since the beginning of 2007. Since 1973, talks have been carried out about airbags becoming usual safety equipment for motorcycles (Honda, BMW) or Scooters (Yamaha) and many prototypes and attempts have been made. However, nowadays there are serious talks about the passive safety in the medium sized motorcycle category. It is sad to say that the development of the passive safety for this category is stagnant. Critics argue that the costs of these developments are not recurrent and it is obvious that there is space for development within this range when it comes to Diploma theses and doctoral works at Universities. The Czech technical university in Prague (CTU in Prague) already offers long term student exchanges and co-operates with the University of Applied Sciences in Dresden (FH) especially in connection with passive safety and biomechanics. Through the materials gathered from both Universities all their experiences concerning passive safety of motorcycles into were summarize into a Ph.D. project. The principle purposes of this doctoral work were defined as follows:

1. reducing the injuries of two-wheeler drivers

2. measurable improvements in the medium sized motorcycle category

3. optimizing the kinetics of the legs and spine the motorbike driver in accident situations

4. conceiving the motorcycle airbags for the protection of the driver's upper body

5. optimizing the airbags by use of virtual and experimental simulations for the minimization of the peak load of motorbike driver

To support these assumptions three motorcycle crash tests into the side of a car were carried out. Statistics show that it is one of the most frequent types of accident. In all of the crash tests the motorbikes were occupied by one passenger in a standard sitting position. The motorcycles differed in the type of sitting position. The following types are concerned: traveling and two extreme (super sport and chopper). The crash tests speed was approximately $60 \mathrm{~km}$ per hour. 
It was clear from the beginning that this work cannot produce results for an optimal solution without the support of a motorcycle producer and a development company for passive safety. Thus since last year the CTU in Prague has co-operated with the local motorcycle producer JAWA and HTW in Dresden, which in turn already co-operates with DELPHI in Berlin, for diploma theses in common. Owing to these possibilities we were able to unify and optimize crucial ideas in our developments at CTU in Prague, especially with the support of these two co-operating partners from a practical point of view. It is also important to note that it was possible to use just some parts of the test results of different institutions (i.e., DEKRA), although without any factual means for our research. Medium sized motorcycles are frequently tested for passive safety, although because of the cost and complicity the result are never implemented in real production

In the year 2003 motorcycle research on accidents began at CTU in Prague with crash-tests and accident simulations for medium sized motorcycles. The Czech technical university had already accomplished many crash-tests. The most frequent kind of accident with fatal consequences for the motorbike driver is through the impact of the motorcycle driver's head to the side or tail of a passenger car. The accident research progressed with the support of investigations through mathematical simulations, as well as real crash tests in development airbags in accident configurations, according to the ISO 13232 (front: 114. side: 413 and back: 711). For the real crash tests we used the ÚSMD dummy, and made some further necessary improvements so that it could be similar to that of a motorbike driver. As for the mathematical simulations, we used the Hybrid III $50^{\text {th }}$ percentile male.

\section{THE INFLUENCE OF THE MOTORCYCLE'S TANK ON INJURY}

Nowadays the motorcycle's tank design has a great influence on the overall design of motorcycle. This has given rise to all sorts of designs and shapes, which in no cases fuse with the passive safety viewpoint. Missing legislations for the protection of motorcyclists by passive safety components make different concepts possible, which do not take into account a motorcyclist's passive safety. In the Transportation Faculty of Transportation sciences a great amount of experimental tests were carried out in which motorcycles of different classification, size, weight, and type of sitting were tested. During the crash tests it was observed relatively often that small deformations were found on the tank. These deformations were caused by the impact of the motorcyclist's pelvic area to the tank.

During the last crash tests in "Faculty of Transportation" sciences, large deformations on the tank in almost all of the performed experiments were discovered. An anthropometric device used for these crash tests has not the appropriate biomechanical properties - biofidelity - in pelvic area. This fact can influence the total deformation of tank. The relative deformation of different types of tanks is comparable. Although this area doesn't cover problems with biomechanical criterions and the standards of passive safety, this deformation in previous crash tests images were documented and were partially assessed. Impacts with the pelvic area, which are the genital and the upper part of the lower limb area of the pelvis, are not taken into account, neither on default anthropomorphic dummies nor in mathematical modules. This is why it is possible to find great potential in university research in this area. While considering the use of airbags it is primordial to talk about the impact of the pelvic area to the motorcycle's tank, since it is expected that the upper body of the motorcyclist is locked by the airbag, but also that there will be an increase of acceleration in the pelvic part of the motorcyclist. 

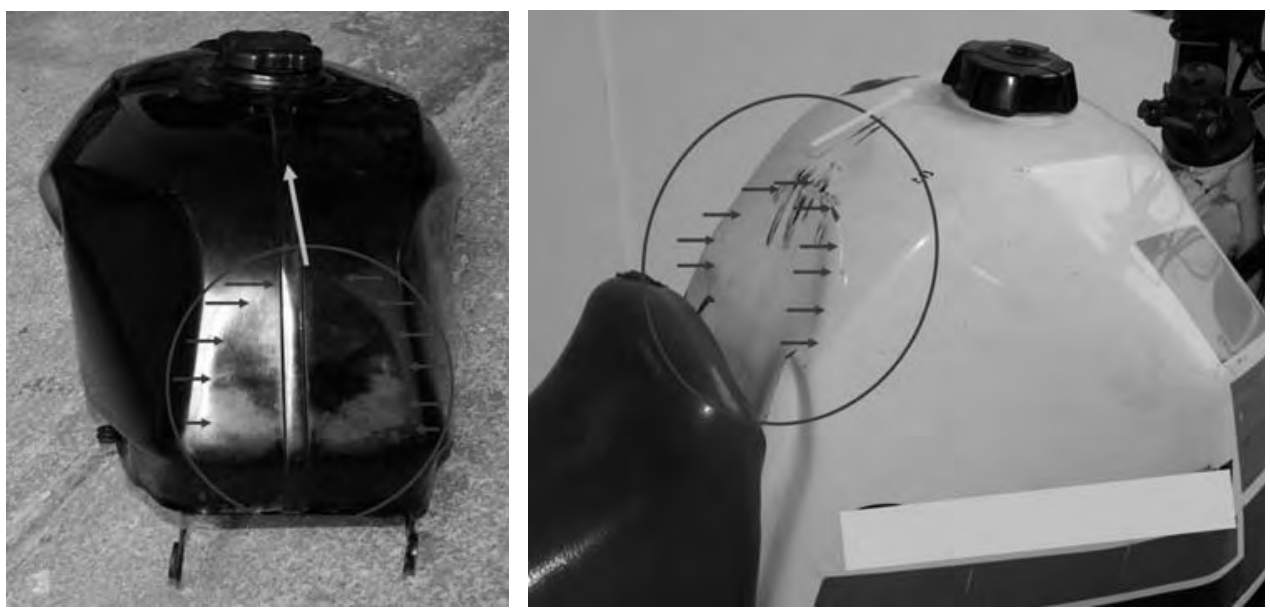

Figure 1a, 1b: Motorcycle tanks after crash tests [3]

The black tank on the left and the white tank on the right are good examples of suitable and unsuitable designs of motorcycle tanks. On the black tank due to the style we can see that it has got a huge deformation by applying a relatively tiny force. On the other hand the deformation on the white tank shows evidence of a great impact and also of a huge damage to the bottom part of dummy's body in the pelvic area.

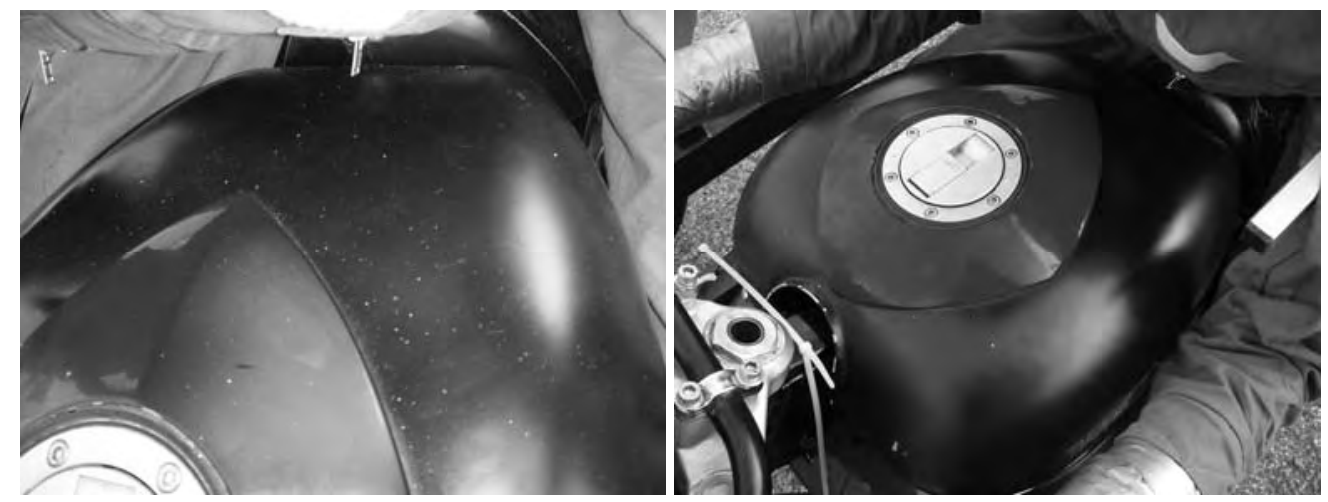

Figure 2a, 2b: Riding setting of motorcyclist and tank in relative location on motorcycle JAWA 650 [3]

However, in general we can conclude that the airbag for motorbike research mentioned in section 1 in CTU in Prague and HTW Dresden is aimed at more factors. These factors are the sitting position, the closing speed, and the shape of a tank. The key aspects are the position of the occupant and the tank. If the airbag is impacted during the collision by the occupant directly, then the airbag efficiency is sufficient. The problem is the position of the occupant and the airbag in the indirect collision, although the task of this research is the comparison of the crash tests with different sitting positions and tank shapes with use of one dummy. On the base of this research a mathematical model of an airbag on the tank in the shape of a letter $\mathrm{T}$ is made.

\section{STANDARD POSITION SETTING OF THE MOTORCYCLIST IN RELATION WITH THE MOTORCYCLE TANK}

The motorcyclist is sitting on the saddle of a motorcycle in the front area. He almost encircles the tank of motorcycle with his legs. The muscles of the pelvic floor are in direct contact with 
the leather foam lug of the front end of the saddle. Femurs of the dummy are in direct contact with the motorcycle's tank. The motorcyclist grips the steering handlebar with both hands. The rest of the arm is free and close to the body and to the motorcycle tank.

\section{ANALYSIS OF THE MOTORCYCLE'S TANK DEFORMATIONS}

We can see a few areas of deformation on the tank after the crash. We could separate these deformations into two parts, the deformations caused by upper part of motorcyclist's femur (area A, marked blue in the illustration), and the deformations caused by motorcyclist's body caused by the pelvic part and by upper part of motorcyclist's femur near coxa (area B, C, D, marked red in the illustration). The green arrows demonstrate the direction of the motorcyclist's movement during the crash.

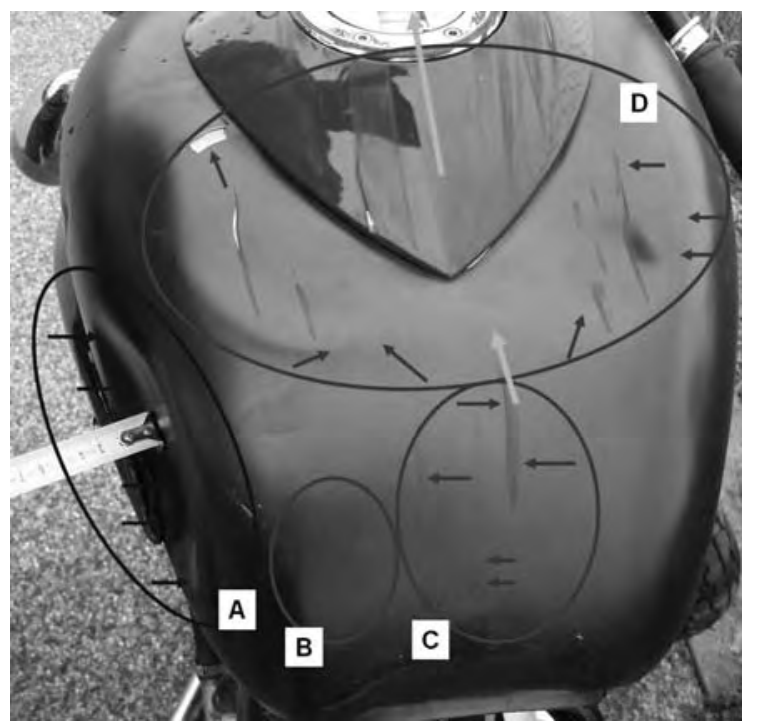

Figure 3: Deformation area on motorcycle JAWA 650 [3]

Deformation in area A was caused by the impact of the upper part of the femur with the motorcycle's tank. The depth of this deformation is $1.7 \mathrm{~cm}$. (Kramer, 2006) This gives evidence of the magnitude of the impact. It is possible to make the assumption that this impact caused trauma in the coxal or femoral area or lower part of the abdomen of a great magnitude. Even if the femur and coxa were able to transfer sizable power, the impact guided in a different angle greater than $90^{\circ}$ to the femur could have worse results. This means that the magnitude of the force is so great that it is responsible for the dislocation of the coxa. As a simple statement of reason of this premise, we could say that for humans the skeleton is more naturally inclined to take the impulsion of force in the vertical plane, because it is built for walking, running, jumping etc., as compared to forces coming from horizontal directions. Also, it is necessary to note that in fact the probable causes of this deformation trauma are influenced by the viscosity in the bottom part of the body.

Deformations in area B are visible only in illustrations 3 and 4a. It indicates small strokes of the upper part of femur between the primary and secondary impact. It is rather probable some overlapping of the left leg under the motorcycle has, as a consequence, an actual deformation of the foot and a fracture of the ankle. From a biomechanics point of view the deformation in the area $\mathrm{B}$ is not a direct cause of any trauma.

In area $\mathrm{C}$ we can see deformations and scratches caused by the motorcyclist's motion on the motorcycle during the front impact in the axis $\mathrm{x}$. This quite tiny deformation was 
caused by the sliding of the pelvis onto the tank. In the case of a human or more exact model of motorcyclist we can speak about the impact of the os ischii and the os pubis and the impact of the musculi perieni. The size of this deformation bears testimony to the suitable design and shape from a passive safety point of view. Although the deformation in this area is not so significant, it proves that in the case of an accident with other motorcycles with different types and shapes of tanks, this deformation could have great significance on the rise of traumas in the pelvic area.

We can assess area D, which is a great deformation zone in the area of the tank's upper surface, as a great impact where the motorcyclist's lower part of abdomen and pelvis sits on the tank. The deformation is marked out by the described ellipse $250 \mathrm{~mm}$ and $150 \mathrm{~mm}$ and a depth of almost $17 \mathrm{~mm}$ and is a testimony to the massive impact. In the case of a human or more exact model of a motorcyclist we can speak about an impact of the lower abdomen (muscles of lower abdomen), os ischii and os pubis and an impact of the musculi perieni.
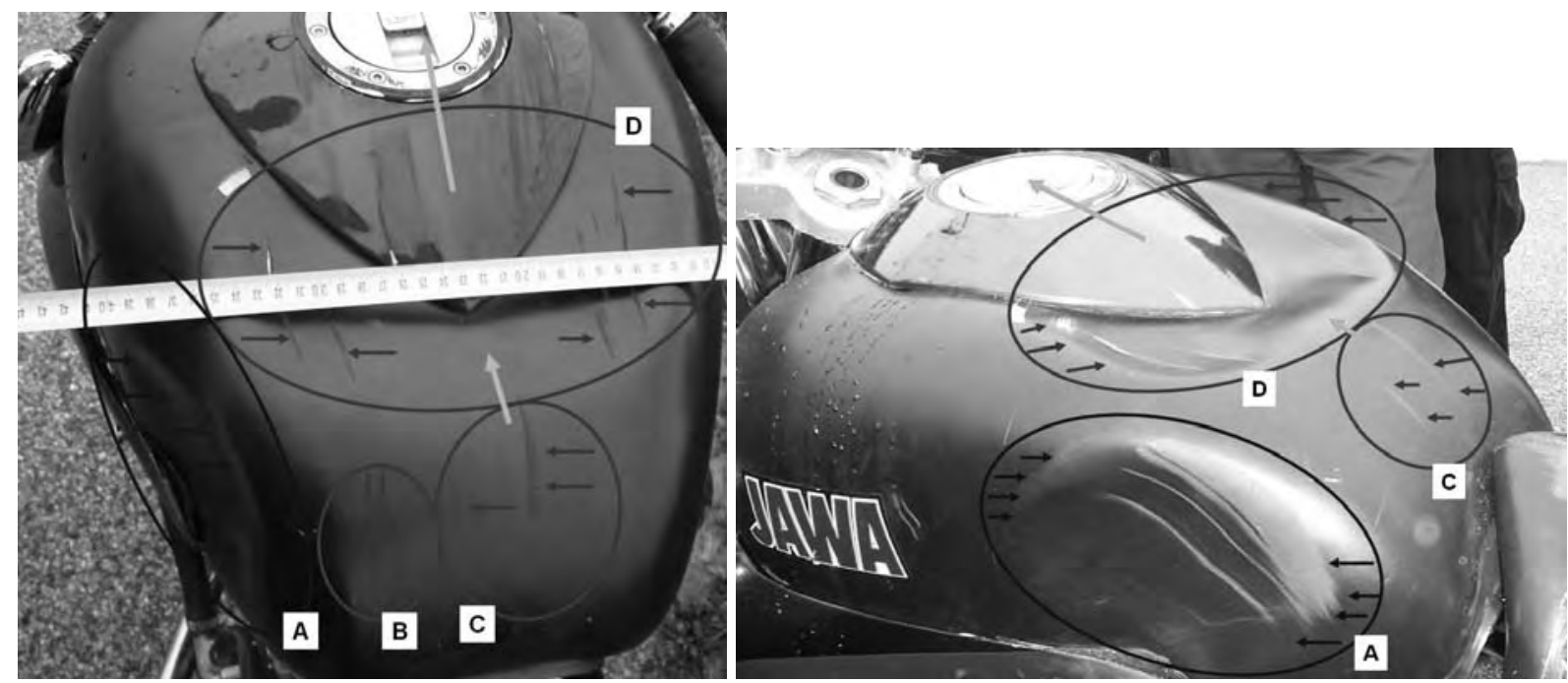

Figure 4a, 4b: Deformation area on motorcycle JAWA 650 - another view [3]

\section{INTERPRETATION OF THE DEFORMATION ON THE TANK OF THE MOTORCYCLE PROTOTYPE JAWA 650}

To separate the deformations and their sizes it is necessary to point out that it isn't possible to determine whether this whole deformation has arisen only during the primary impact or only during the secondary impact. When talking about the impact of the motorcycle to the lying motorcyclist or the direct pinning of the motorcyclist considering the projection. It's very likely that the deformation given arose during the primary impact and has been only partly deepened during the secondary impact. Reading the memory of the high speed camera and the data from the sensors we can assume that during the secondary impact no further tank deformations occurred, but we cannot exclude the contact possibility completely.

The implication of these deformations has effects on human trauma (in our case trauma of motorcyclist) each time after an accident. From the passive safety point of view we can say that there are critical deformations in area A and D. We can consider deformations B and $\mathrm{C}$ as almost insignificant, although deformation $\mathrm{C}$ holds a great potential for research. 

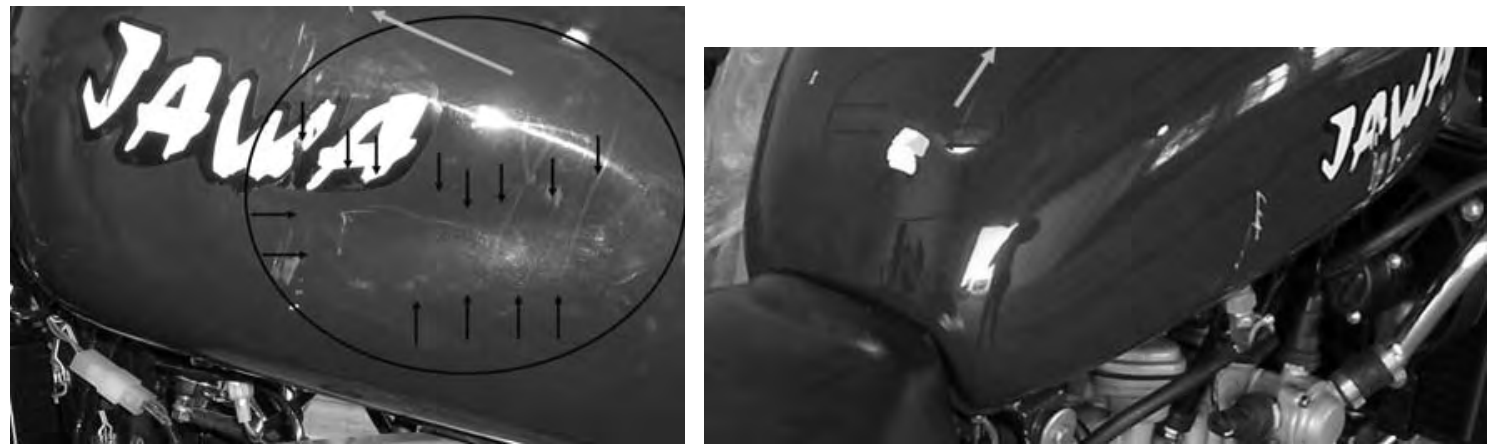

Figure 5a, 5b: Deformation area on motorcycle JAWA 650 Classic [3]

Between the motorcycle's tank deformation of Jawa 650 Classic and Jawa 650 (Bison) it is possible to see a huge difference in deformations after the crash test under the same conditions, speed and with the same dummy. This means that they are almost the identical tanks (Bison has in addition plastic cover under the tank cover), but with absolutely different deformations. It is possible to explain this difference by the motorcyclist's sitting position, because the motorcycle Jawa 650 (Bison) - as the prototype for passive safety testing, was set up in the position of steering handlebar and crampons as a motorcycle of the Super Sport type. The common series of the Jawa 650 Classic model has succeeded completely without any trauma during the crash testing of tank. We would like to express our appreciation to the Jawa company for supporting our research and for altering their designs so as to maximize passive safety on their motorcycles.

In general we can conclude that these assumptions were made after a subjective evaluation of the trajectory of the passenger, recorded by high speed camera, the size of deformation of tank, and with objective data from the accelerator in the chest area. On the basis of this subjective assumption the following shapes of tank were chosen as examples of subjectively potential suitable and unsuitable motorbike tank shapes.
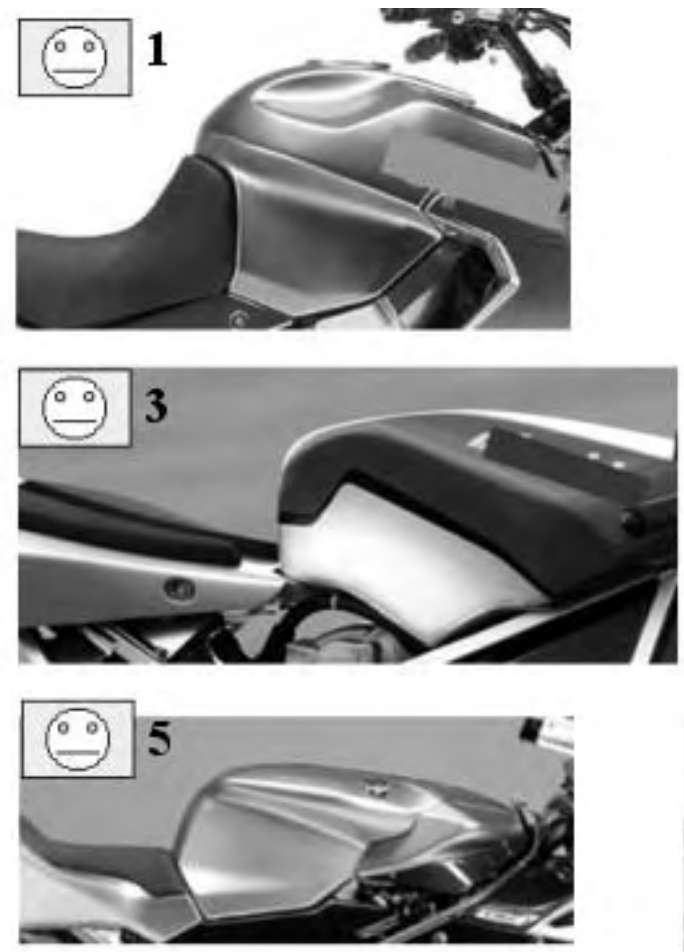
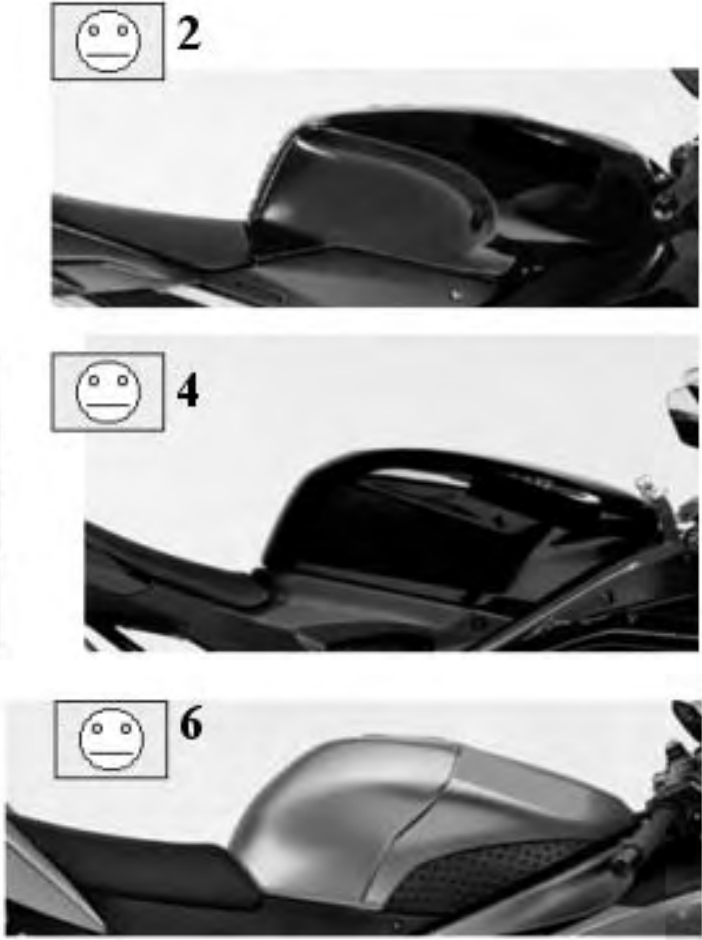

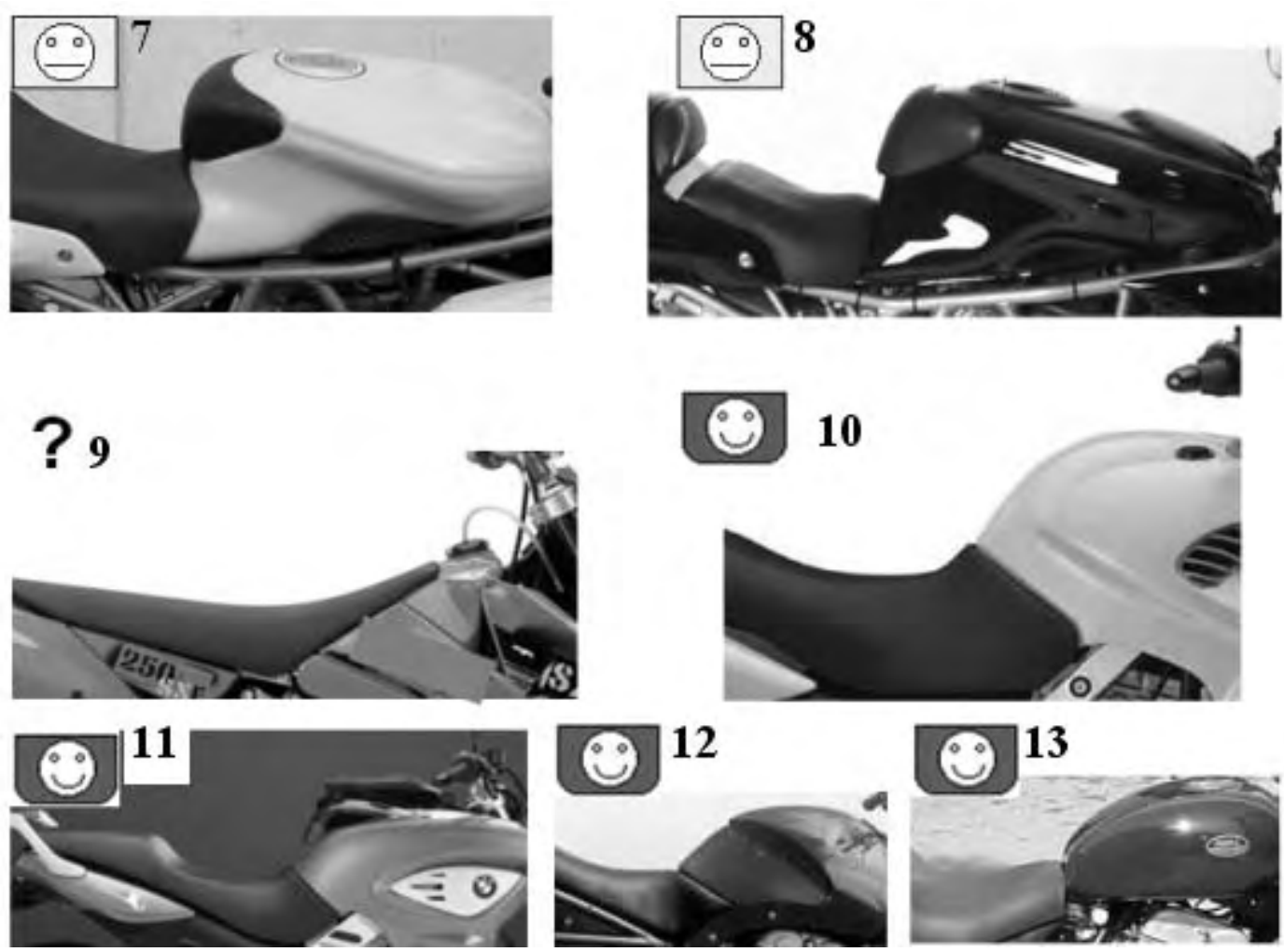

Figure 6: Some shapes of motorcycles tanks [8]

On the previous pictures different types of tanks from different types of motorcycle are shown. Each picture is assigned a smiley that represents the possible injury risk of various motorcycle tanks. It is comprehensible that it is only a subjective opinion at the moment and that it would be necessary to evaluate them using a significant number of crash tests and computer simulations. In spite of this, it is possible to decide from the naked eye, whether the set concept fuses with the unwritten rules of passive safety.

Tank No. 1: The shape indicates the intention of the constructor to project the driver over the contingent collision partner, but the ergonomic embrasure spaces contradict this initial intention.

Tanks No. 2, 4, 5 \& 6: None of the shapes, nor the angles that the tanks make with the driver's seat nor the ergonomic embrasures of these tanks correspond even remotely to any passive safety point.

Tank No. 3: This shape is the worst of them all as it will prevent the driver's projection from the motorbike causing considerable damage in the pelvic area.

Tank No. 7: This shape has neither any direct influence in injuring the driver nor does it have any influence in slowing the driver's projection, but instead the driver is projected directly onto the steering handlebars.

Tank No. 8, 9, 10, 11: These shapes influence the driver's projection trajectory and are probably not the cause of any serious injuries to the driver.

\section{INFLUENCE OF TANK ON PASSIVE SAFETY OF MOTORCYCLE}

From an evaluating point of view it is possible to claim that it should be at most suitable to open a discussion about giving rise to a new criterion in motorcyclist's coxa area trauma during the impact with the motorcycle tank. The eventual criterion should specially consist 
in the integration of different methods of research and obtaining necessary data, whether it be from medicine, passive safety, biomechanics or from the development of possible motorcycle's airbag and its application. It is essential to conduct further research connected with the impact of the pelvic area and tanks, without which the implementation of airbags on motorcycles would be completely redundant.

\section{IMPLEMENTATION OF THE AIRBAG ON THE MOTORCYCLE TANK}

The function of the common car airbag as part of the passive safety support system could not be converted directly for classical or large motorcycles, because the motorcycle driver does not have a definite sitting position, just as it is in the case of the passenger in the car. In addition for the motorcycle airbag, the influence of the seating position, as well as the motion path, must be considered during frontal collisions. CTU in Prague, in cooperation with HTW Dresden, has concerned themselves with the deepening of vehicle safety in the department of vehicle technology since 2004, including the sitting position, tank shape (as mentioned above) and the seat kinematics of the motorcycle passenger (Kramer, 2006). The driver position could be divided into 12 main groups of motorcycles and could be divided further into static or dynamic driving positions (traveling straight ahead, driving along curves). Static driver position means traveling straight ahead without pre and back bends. Dynamic position means driving along curves, as well as traveling straight ahead with pre and back bend. The driver position plays an extremely important role for the use of airbags on the motorcycle tank.

Unfavorable sitting positions of the driver, which prevents an optimal use of the airbags, must also be considered with the organization and tuning of the airbags to an extent where at least endangering of the driver must be excluded! Some years ago airbags for motorbike driver began with the commercial offer of the "airbag jacket", an airbag, which was integrated in a jacket or waistcoat of the motorbike driver. A motorcycle airbag should with 90 litres of gas volume protect from the moving of the chest and neck range. The release was made by activation-line and offered rather as a protection against secondary collision with the roadway. The first series use of the airbags on the motorcycle was done by Honda in the year 2007. The motorcycle Honda Gold Wing uses sensor systems of the collision and is activated by the same acceleration sensors which are placed on the front fork. In the cockpit of the motorcycle instead of a tank an airbag generator is placed, similar to the passenger car. During the collision phase the driver is caught by the motorcycle airbag, which is embodied and supported by special straps on the vehicle's frame. This airbag has to protect against structures of the collision partner or to reduce the contact speed in a secondary crash. The conversion of this airbags for large motorcycles with relatively defined seating position is possible. But the question is how can this principle be transferred to middle class motorcycles?

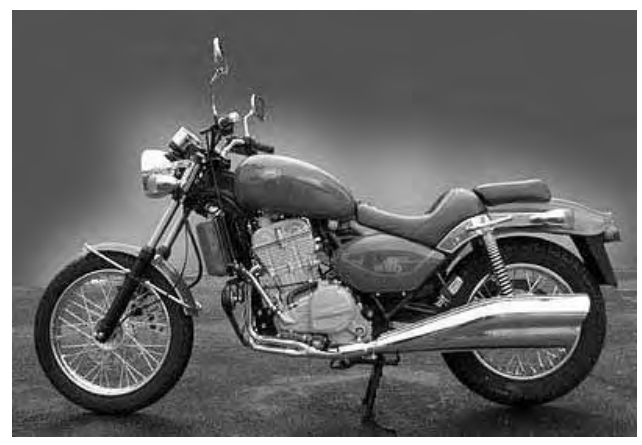

Figure 7: Motorbike Jawa 650 Classic [2] 
The greatest portion of the work to be done is that which is connected with experimental simulations, which were accomplished at CTU (First \& Kovanda \& Lenk, 2004). The results of the experimental simulations made virtual simulations possible and obtained data were also used for validation.
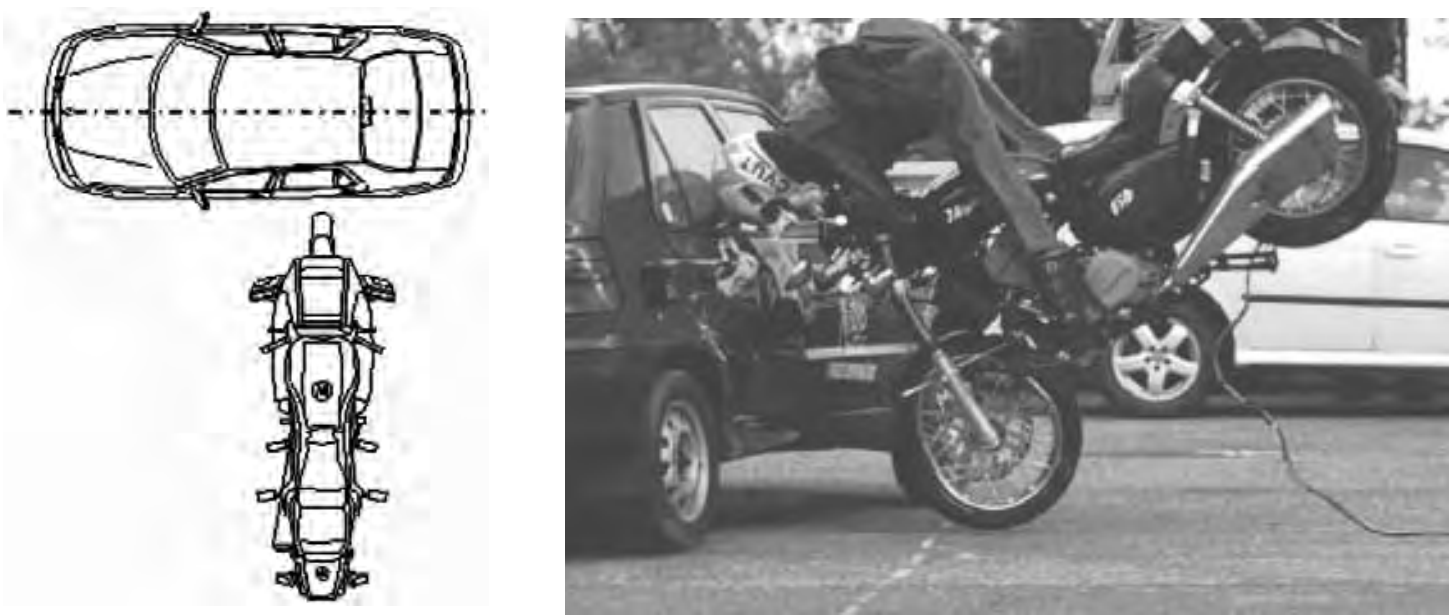

Figure 8a, 8b: Experimental simulation in impact configuration 413 according to ISO 13232 [2]

For validating accelerations in the head and in the chest dummies were used and the tensions and deformations in the frame of the motorcycle were monitored. The film from the real collision was also used for the evaluation of the flight path of the motorbike driver for simulation purposes. The virtual simulation of the passenger car/motorcycle collision depends not only on the experimental data, but also on the particular technical data of the passenger car and the motorcycle. We first simulated using the computational configurations without safety precautions and without an airbag in the collision types 115, 413, 711 according to ISO 13232 for Car/Motorbike accidents.

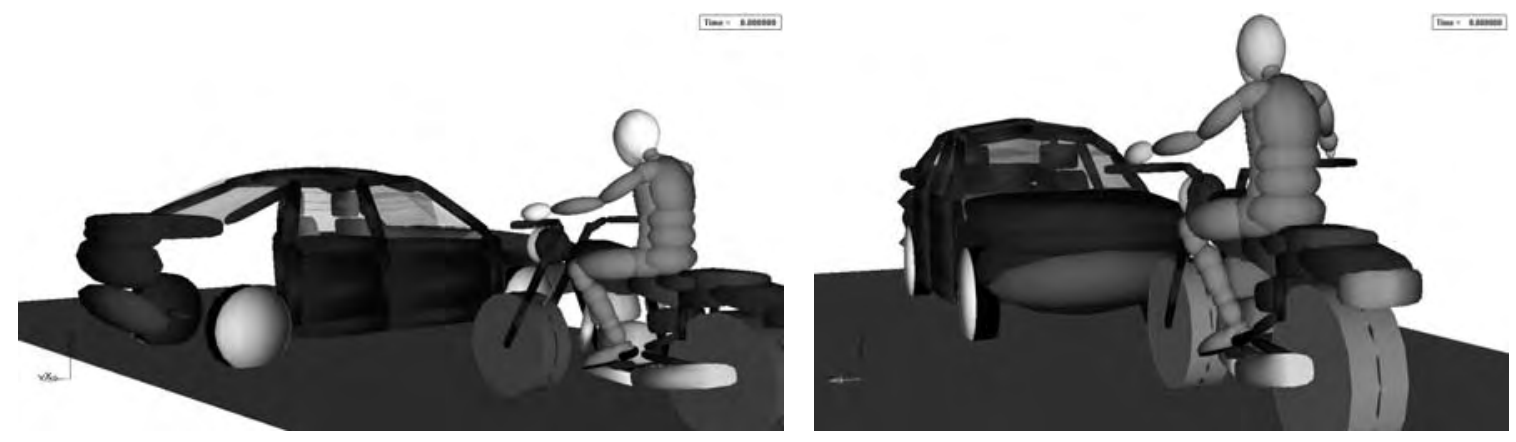

Figure 9a, 9b: Computer simulation without airbag in impact configuration 115 and 413 according to ISO 13232

The next computer simulation was accomplished with a standard passenger car front seat passenger airbag of 150 litres in volume. Furthermore possible deformation structures were optimized like the front fork and further measures for improving the passive safety (e.g. employment of a Knee airbag) were tested and through this the most suitable one was selected. In the revised virtual simulations we discovered that the 115 litres full volume Tformed airbag was the most effective passive safety measure possible. This airbag was also tested in the out of position situations. It's special form and size clearly made it possible to have more favorable results than the original (front seat passenger airbag). 


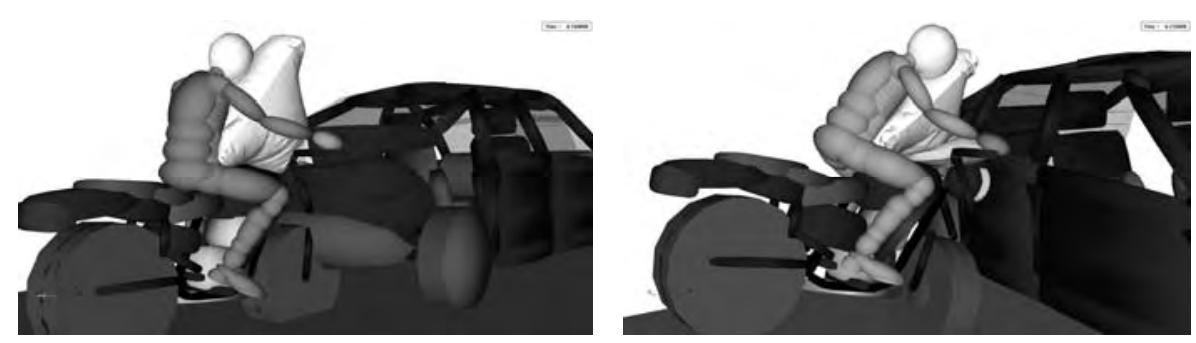

Figure 10a, 10b: Computational simulation with airbag (2.Generation), deformation structure, Knee airbag in impact configuration 711 and 413 according to ISO 13232

The improved results refer to the values of HIC, T3MS, FFC, after the data acquisition, comparison and validation by virtual and experimental simulations.

\section{ACKNOWLEDGMENT}

The work was supported by the grant of Ministry of industry and trade "Application of engineering mechanics and biomechanics in transport for the improvement of passive safety and comfort of occupants", ID: FT-TA/024.

\section{SUMMARY}

By employing the experimental and virtual simulations for the gradual optimization of the construction and development of a special motorcycle airbag with appropriate structural changes to the motorcycle, clearly improved results in decreasing of injury sequences for the motorbike driver with continuous accident weight were confirmed. This particularly applies to it's use with motorcycles of the middle class, where substantially even more different driver positions are possible than with the heavy route machine of Honda "Gold Wing", which exhibits the only series-solution of this support mechanism for motorcycles at present.

\section{REFERENCES}

[1] Berg \& Rücker, 2004. Prüfverfahren für die passive Sicherhiet motorisierter Zweiräder (Testing method for the passive safety of motorized two-wheelers). Study, Stuttgart.

[2] First \& Kovanda \& Lenk, 11/2004. Kolize motocykl - automobil (Motorcycle-car collision), Technical report, VYZ616.003/04, Prague, FD CTU.

[3] First \& Kovanda \& Šotola, 12/2007. Kolize motocykl - automobil (Motorcycle-car collision), Technical report, VYZ616.005/07, Prague, FD CTU.

[4] Kovanda \& Šatochin, 2000. Pasivni bezpečnost vozidel (Vehicles pasive safety). University mimeographed, Prague, CTU.

[5] Kramer, 3/2006. Passive Sicherheit von Kraftfahrzeugen (Passive safety of motor vehicles), University book, Dresden.

[6] 2005-6, Seminární práce na HTW Drážd’any z předmětu odborné znalecké vědy, Vorträge Sachverständigenwesen an der HTW Dresden, (Lectures expertises on the HTW Dresden).

[7] Výkresové materiály firmy Jawa a.s. (Technical drawing of motorcycle company JAWA), [CD-ROM].

[8] www.motocykl-online.cz 